\title{
Surface Verwey Transition in Magnetite
}

\author{
Zbigniew Łodziana* \\ Institute of Nuclear Physics, Polish Academy of Sciences, ul. Radzikowskiego 152, PL-31-342 Kraków, Poland \\ Department of Environment, Energy and Mobility, EMPA, 8600 Dübendorf, Switzerland
}

(Received 9 May 2007; published 13 November 2007)

\begin{abstract}
We report density functional studies of the (001) surface of magnetite that account for local Coulomb interactions. Iron cations in the surface layers exhibit charge and $t_{2 g}$ orbital ordering that is coupled with the lattice strains. Orbital ordering is present for various surface stoichiometries and causes opening of the band gap $E_{g} \sim 0.3 \mathrm{eV}$ at the surface, such that the (001) surface of $\mathrm{Fe}_{3} \mathrm{O}_{4}$ remains insulating also in the high temperature cubic phase. The $(\sqrt{2} \times \sqrt{2}) R 45^{\circ}$ surface reconstruction is related to orbital ordering.
\end{abstract}

DOI: 10.1103/PhysRevLett.99.206402

PACS numbers: 71.30.+h, 68.47.Gh, 73.20.At

In 1939 Verwey described the metal-insulator transition in magnetite, $\mathrm{Fe}_{3} \mathrm{O}_{4}[1]$, lodestone, known already to medieval sailors or even Olmecs [2]. Despite enormous research efforts, not all mysteries of this material are known today $[3,4]$ and Verwey's discovery is still debated [3-5]. While some agreement about nature and the origin of the metal insulator Verwey phase transition in the bulk $\mathrm{Fe}_{3} \mathrm{O}_{4}$ seemed to emerge recently [6,7], properties of the magnetite surface are still puzzling $[8,9]$. This especially concerns the hypothesis that the surface Verwey transition occurs at temperature much higher than $T_{V} \sim 125 \mathrm{~K}$ for the bulk $[9,10]$. The other debated problem concerns explanation of the observed $(\sqrt{2} \times \sqrt{2}) R 45^{\circ}$ reconstruction of the (001) surface [11]. Below we propose the explanation of both problems related to the (001) surface of $\mathrm{Fe}_{3} \mathrm{O}_{4}$.

Above the $T_{V}$ magnetite is a ferrimagnet that crystallizes in the inverse spinel structure $(F d \overline{3} m)$. High critical temperature $\left(T_{C} \sim 860 \mathrm{~K}\right)$ makes it interesting for magnetic and highly sensitive spintronic devices. In the spinel structure iron cations occupy tetrahedral $\mathrm{Fe}(A)$ and octahedral $\mathrm{Fe}(B)$ interstices. At room temperature magnetite is a poor metal with electronic conductivity resulting from the minority $t_{2 g}$ orbitals of $\mathrm{Fe}(B)$ cations that cross the Fermi level. In the traditional picture the $\operatorname{Fe}(A)$ sublattice is occupied by $\mathrm{Fe}^{+3}$ cations while the $\mathrm{Fe}(B)$ sublattice consists of a randomly distributed equal number of $\mathrm{Fe}^{+3}$ and $\mathrm{Fe}^{+2}$ cations. A drop of the conductivity at the Verwey transition was originally ascribed to the particular charge ordering of the octahedral $\mathrm{Fe}(B)$ sublattice $[1,3,5]$. Recent studies, however, question this explanation [4]. There is a common agreement that the valence charge difference on various $\mathrm{Fe}$ cations does not exceed a few tenths of $e$ $[4,12,13]$, and a subtle orbital ordering among octahedrally coordinated Fe occurs [6,7]. The mechanism of the orbital ordering is related either to the coupling of electrons to the lattice distortion $[6,7,14]$ or to the dimerization between $\mathrm{Fe}$ [15]. Whichever the origin of the metal insulator transition is, it is clear that electron-lattice coupling and the local Coulomb interactions between Fe $d$ electrons play a crucial role in understanding the Verwey transition [16].
The intensive studies of the surface of $\mathrm{Fe}_{3} \mathrm{O}_{4}$ date from the very first STM experiment [10] with this material. For the surface studies of $\mathrm{Fe}_{3} \mathrm{O}_{4}$ two experimental procedures are common: cleavage of single crystals $[9,10]$ and growth of epitaxial films $[8,11,17]$. Both methods require surface preparation that occurs over the broad range of temperatures and pressures; thus difficulties in controlling particular surface stoichiometry are common. Irrespective of the surface preparation method the (001) magnetite surface reconstructs to $(\sqrt{2} \times \sqrt{2}) R 45^{\circ}$. The $(001)$ termination of the $\mathrm{Fe}_{3} \mathrm{O}_{4}$ is polar $[8,18]$. Consequently, a mechanism of the surface charge compensation is usually discussed together with various structural models of the surface reconstruction. Structural models of the reconstructed $(\sqrt{2} \times \sqrt{2}) R 45^{\circ}(001)$ surface include those with $\mathrm{Fe}(A)$ termination, where half of surface cations are missing [11] or models with $\mathrm{Fe}(B)$ termination with oxygen vacancies or hydroxyl groups on the surface [17]. The surface stability can also be achieved via modification of electronic degrees of freedom, and appropriate models for octahedral termination with Jahn-Teller lattice distortion were proposed [19]. Already in the early 1990s it was suggested that the surface charge ordering on $\operatorname{Fe}(B)$ may occur at temperatures higher than $T_{V}$ [10] and only recent room temperature STM experiments showed direct evidence that the surface of magnetite is indeed insulating and it has a band gap of $0.2 \mathrm{eV}$ [9]. This stands in conflict with theoretical findings of surface metallization [19].

In this Letter we report density functional theory (DFT) studies of the (001) magnetite surface accounting for electron correlations via on-site Coulomb interaction $U$ and exchange parameter $J$ [20]. This approach was very successful for the bulk material [6,7]. Our studies shed new light on controversies about this surface, providing an indication that the surface lattice distortion coupled to the ordering of $\mathrm{Fe} d$ orbitals leads to the surface metal insulator transition, and the surface remains insulating also in the high temperature cubic phase of $\mathrm{Fe}_{3} \mathrm{O}_{4}$. Additionally, we argue that the orbital ordering is responsible for the surface reconstruction. 
We have used a periodic DFT approach [21], where the surface was represented by a symmetric slab with 15 atomic layers $(\sim 100$ atoms $)$ and $12 \AA$ of vacuum. The $(\sqrt{2} \times \sqrt{2}) R 45^{\circ}$ surface unit cell was considered. The valence configuration $3 d^{7} 4 s^{1}$ for $\mathrm{Fe}$ and $2 s^{2} 2 p^{4}$ for $\mathrm{O}$ were represented by projected augmented wave potentials [22]. An energy cutoff of $400 \mathrm{eV}$ and a $4 \times 4 \times 1 k$-point grid were applied. The generalized gradient approximation for the exchange-correlation functional [23] and Coulomb $U=4.5 \mathrm{eV}$ and exchange $J=0.89 \mathrm{eV}$ parameters were used [24]. The ground state electronic density was determined by iterative diagonalization of the Kohn-Sham Hamiltonian, and the self-consistent procedure was applied to the GGA $+U$ Hamiltonian $[20,25]$ (GGA denotes the generalized gradient approximation) until convergence of the occupation numbers and total energy [25] was achieved. Then forces on atoms were calculated and each surface was relaxed until they were smaller than $0.02 \mathrm{eV} / \AA$.

In the present studies we have considered most of the standard (001) $\mathrm{Fe}_{3} \mathrm{O}_{4}$ surface models: terminated with full tetrahedral $\mathrm{Fe}(A)$ or octahedral $\mathrm{Fe}(B)$ layer, half-filled tetrahedral layer $[0.5 \mathrm{Fe}(A)]$; octahedral termination with oxygen vacancy $\left[\mathrm{Fe}(B)+\mathrm{V}_{\mathrm{O}}\right]$. Additionally termination with tetrahedral $\mathrm{Fe}$ cations forming a dimer [8] was considered, denoted as $\operatorname{Fe}(A) d$. We use this short notation to the end of this Letter.

The surface phase diagram $[26,27]$ is presented in Fig. 1. It agrees with this based on standard DFT and reported previously $[19,28] ; \mathrm{Fe}(B)$ termination is the most stable over a broad range of oxygen potential. Significantly smaller differences between surface energies result from orbital (and charge) ordering on the surface cations, which will be discussed below.

We note that for $0.5 \mathrm{Fe}(A)$ surface tetrahedral cations do not penetrate into the octahedral layer as reported by models that do not account for electron correlations $[11,19]$. The lowest energy configuration for $\mathrm{Fe}(A)$ termi-

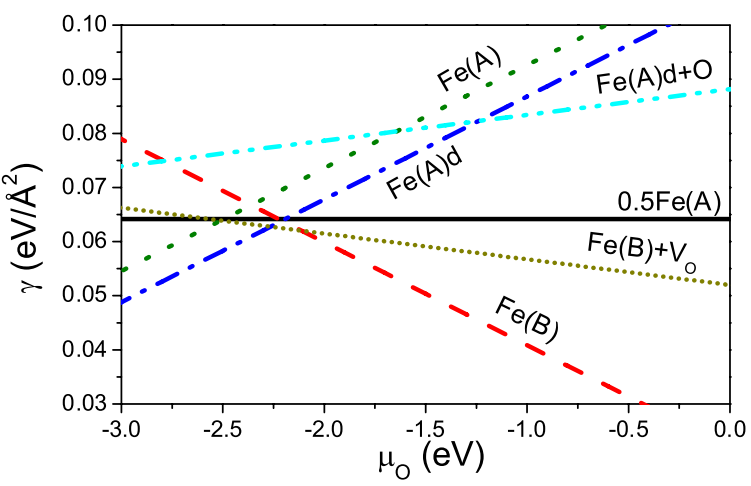

FIG. 1 (color online). Surface energy diagram for the (001) face of $\mathrm{Fe}_{3} \mathrm{O}_{4}$. The UHV conditions correspond to $\mu_{\mathrm{O}} \approx-2 \mathrm{eV}$. Symbols are explained in the text, and $\mathrm{Fe}(A) d+\mathrm{O}$ stands for the oxygen atom adsorbed on the surface tetrahedral iron. nation is the surface where cations are separated by $4 \AA$, ca. $2 \AA$ closer than their original bulk position [denoted as $\operatorname{Fe}(A) d$ in Fig. 1]. Surface cations are located such that subsurface tetrahedral $\mathrm{Fe}$ is located below the dimer center. This configuration is correlated with orbital ordering on the first $\operatorname{Fe}(B)$ layer.

One has to keep in mind that surface stability, as shown in Fig. 1, concerns thermodynamics, and on this basis none of the surface terminations can be excluded in the conditions of UHV experiments $\left(\mu_{\mathrm{O}} \sim-2 \mathrm{eV}\right)$.

To reveal the mechanism beyond surface relaxations now, we turn our attention to structural and electronic modifications of the surfaces. These modifications originate from reduced dimensionality of the system. They can be seen as the competition between elastic and charge compensation effects. The latter consists of various factors: the primary one, which is related to the charge compensation due to reduced coordination of surface atoms and/or stabilization of the polarity. In materials with strong electron correlations, the second effect is related to charge (orbital) ordering coupled to the lattice distortion. The primary effect of charge compensation is present for all metal oxide surfaces. In the case of polar terminations this effect can lead to the surface reconstruction or even to the surface metallization [29]. In magnetite the local Coulomb repulsion of $d$ electrons leads to the opposite phenomenon-opening of the band gap, as we explain below.

The interlayer spacing between atomic layers parallel to the surface for the $\mathrm{Fe}(B)$ termination of $(001) \mathrm{Fe}_{3} \mathrm{O}_{4}$ changes by $\sim-8 \%$ for the first and second $\mathrm{Fe}(B)$ layers and by $\sim+5 \%$ for the second and third layers. Additionally, broken $\mathrm{FeO}_{6}$ octahedral symmetry at the surface removes degeneracy of $e_{g} \mathrm{Fe}(B)$ orbitals near the surface. The $\mathrm{Fe}-\mathrm{O}$ bond length decreases from $2.05 \AA$ in the bulk to $1.97-1.99 \AA$ at the $\operatorname{Fe}(B)$ terminated surface plane. The $e_{g}$ bands are located well above the Fermi energy $\left(E_{\mathrm{F}}\right)$, i.e., $d_{z^{2}}$ at $\sim 2.5 \mathrm{eV}$ for the surface $\mathrm{Fe}(B)$; see Fig. 2(a). The occupied states located $1 \mathrm{eV}$ below $E_{\mathrm{F}}$ result from hybridization of $d_{x^{2}-y^{2}} \mathrm{Fe}(B)$ orbitals with nearest oxygen $p$ shells [see Fig. 2(a)]. This orbital overlap contributes to screening [6]. Consequently, minority $t_{2 g}$ electrons (responsible for conductivity of the cubic $\mathrm{Fe}_{3} \mathrm{O}_{4}$ ) are pushed above the Fermi level — thus the band gap opens for the topmost surface layer (see Fig. 2). No orbital or charge ordering is present for the topmost iron cations on the $\mathrm{Fe}(B)$ surface. The density of states for the first subsurface $\mathrm{Fe}(B)$ layer is depicted in Fig. 2(b). Octahedral cations are divided into two pairs: one with $t_{2 g}$ states that are located above the $E_{\mathrm{F}}$, and another pair with minority spin states localized $\sim 0.5 \mathrm{eV}$ below the $E_{\mathrm{F}}$ [see Fig. 2(b)]. The ordered states consist of $d_{x y}$ orbitals oriented in the plane parallel to the surface.

The real space projection of these localized states for $\mathrm{Fe}(B)$ termination is depicted in Fig. 3(a). The $\mathrm{Fe}(B)$ in the subsurface layer are paired, which can be perceived as the 

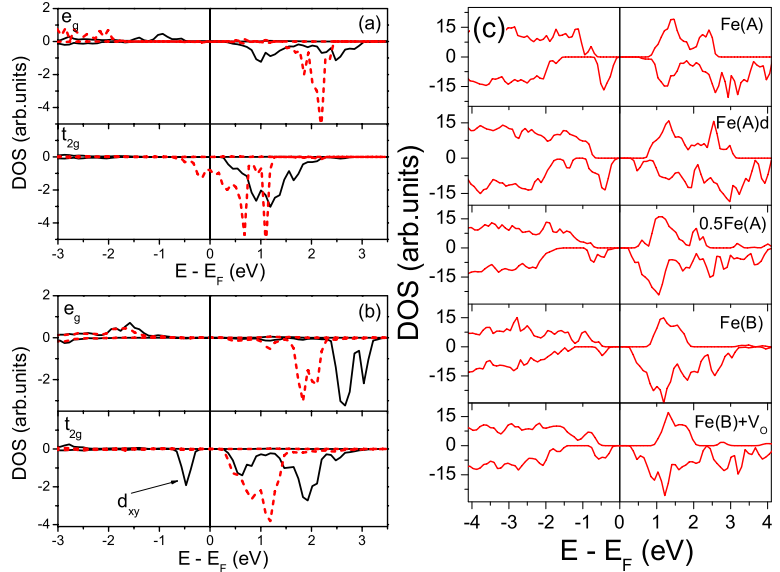

FIG. 2 (color online). Electronic density of states projected on the $\operatorname{Fe}(B) d$ orbitals for the first (a) and second (b) surface layer. Dashed line in (a) represents density of states for the bulk. In (b) dashed and solid lines represent two types of Fe cations. The total density of states of two surface layers and for all models considered in this Letter (c).

Peierls lattice distortion [15]. The distortion of both iron and oxygen sublattices can be seen in Fig. 3(a), and it indicates strong coupling between electronic degrees of freedom and lattice strains. The orbital ordering occurs for cations separated from adjacent oxygen by $2.03 \AA$ to
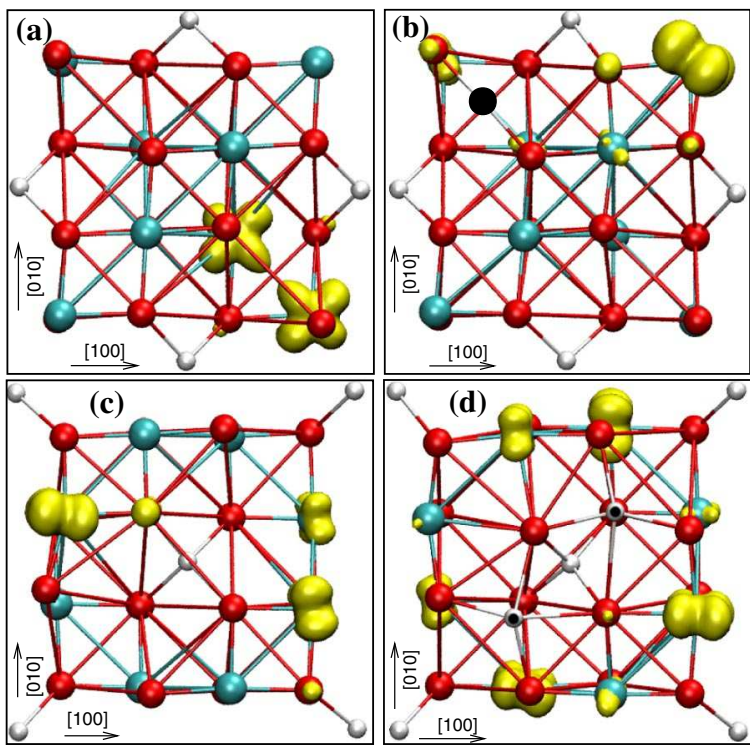

FIG. 3 (color online). Electron density integrated around localized state $E-E_{\mathrm{F}}=-0.5 \mathrm{eV}$ (a) octahedral termination $\mathrm{Fe}(B)$; (b) $0.5 \mathrm{Fe}(A)$ termination; (c) oxygen vacancy on the octahedral termination; (d) tetrahedral termination $\operatorname{Fe}(A) d$. Large (blue) spheres are for $\operatorname{Fe}(B)$ cations, small (white) represent $\mathrm{Fe}(A)$, and medium (red) are for oxygen. Only the two topmost surface layers of the single $(\sqrt{2} \times \sqrt{2}) R 45^{\circ}$ surface cell are shown for clarity. The surface is parallel to the picture plane. $\operatorname{Fe}(A)$ cations above octahedral layer in (b) and (d) are marked with black dots.
$2.17 \AA$. Other pairs of cations reside in more symmetric surroundings with $\mathrm{Fe}-\mathrm{O}$ spacing of $\sim 2.03 \AA$. Remarkably, the lattice distortion with a period of two gives the $(\sqrt{2} \times$ $\sqrt{2}) R 45^{\circ}$ surface reconstruction.

The band gap of $\sim 0.3 \mathrm{eV}$ that opens for two surface layers of $\mathrm{Fe}(B)$ termination described above persists for all other surface stoichiometries considered in the present studies; see Fig. 2(c). The presence of tetrahedral cations or oxygen vacancies on the surface initiates ordering of iron $d$ orbitals also in the upper $\mathrm{Fe}(B)$ layer [Figs. 3(b)3(d)]. Localized states are present for $d_{x z}$ and $d_{y z} d$ orbitals - perpendicular to the surface. This is related to elongation of $\mathrm{Fe}-\mathrm{O}$ bonds and a different surface strain pattern of terminations with a perturbed octahedral layer. The orbital ordering is very similar to the Kugel-Khomskii ground state found for the low temperature bulk phase of $\mathrm{Fe}_{3} \mathrm{O}_{4}$ [6,7]. In general localized states appear for $\mathrm{Fe}$ cations with the longest and most distorted bonds to oxygen anions. Their $\mathrm{Fe}-\mathrm{O}$ separation ranges from $1.98 \AA$ for termination with $\mathrm{V}_{\mathrm{O}}$ at the surface, through $2.02-2.15 \AA$ for $0.5 \mathrm{Fe}(A)$ to $2.18 \AA$ for the $\mathrm{Fe}(A) d$ surface. The orbital ordering in the subsurface layer persists irrespective of surface termination [30].

The local magnetic moments, $\mu$, and the differences of the valence charge $\delta q$ and $t_{2 g}$ orbital occupancy for the Fe cations in the vicinity of the surface are presented in Table I. Similar to the previous reports on the bulk magnetite [31,32], the magnetic moments are reduced for the cations that possess ordered orbitals. The valence charge of these cations is larger than the other pair by $(0.2-0.4) e$, which is slightly more than for the bulk, but can be explained by much larger lattice deformation. The charge ordering can be seen in differences of $t_{2 g}$ orbital occupancy.

The band gap opening and similarities between surface and the bulk local electronic effects on the octahedral iron suggest that the electronic state of the surface resembles this for the magnetite structure below the Verwey transition temperature. In particular, the strong lattice deformation at the surface coupled to the electronic degrees of freedom

TABLE I. Magnetic moments (in $\mu_{B}$ ) and differences of ionic charges and $t_{2 g}$ occupancy (in $e$ ) for the octahedral Fe on the (001) surface of $\mathrm{Fe}_{3} \mathrm{O}_{4}$. For the low temperature bulk phase, the magnetic moments of 4.00, $-3.57,-4.08$ [31] and 3.84, -3.54 , -4.00 [32] were reported for $\mathrm{Fe}(A), \mathrm{Fe}(B)$, and $\mathrm{Fe}\left(B^{\prime}\right)$ cations. For $\mathrm{Fe}(B)$ termination subsurface cations are considered.

\begin{tabular}{lcccccr}
\hline \hline Layer & bulk & $\mathrm{Fe}(A)$ & $\mathrm{Fe}(B)$ & $0.5 \mathrm{Fe}(A)$ & $\mathrm{Fe}(B)+\mathrm{V}_{\mathrm{O}}$ & $\mathrm{Fe}(A) d$ \\
\hline $\mathrm{Fe}(A)$ & 3.95 & 3.95 & 3.97 & 3.96 & 3.96 & 3.96 \\
$\mathrm{Fe}(B)$ & -3.90 & -3.60 & -3.90 & -3.63 & -3.53 & -3.59 \\
$\mathrm{Fe}\left(B^{\prime}\right)$ & - & -3.99 & -4.10 & -4.03 & -4.05 & -4.00 \\
$\delta q\left(B-B^{\prime}\right)$ & - & 0.3 & 0.2 & 0.3 & 0.4 & 0.3 \\
$\delta q\left(t_{2 g}\right)$ & - & 0.64 & 0.65 & 0.62 & 0.73 & 0.64 \\
\hline \hline
\end{tabular}


indicates this coupling is important for the explanation of the Verwey transition.

Recently Jordan et al. [9] reported STM spectra for the (001) and (111) surfaces of the magnetite single crystal, providing direct evidence for the surface band gap of $\sim 0.2 \mathrm{eV}$ that is present at temperatures well above the Verwey transition. In view of our studies, the band gap opening is a natural manifestation of strong electron-lattice strain coupling and the local Coulomb repulsion of Fe $d$ electrons. The structural features like the wave modulation [19] along [110] can also be understood in view of $\mathrm{Fe}(B)$ orbital ordering, but unlike the local spin density approximation method [19] GGA $+U$ gives the insulating surface.

The charge ordering of the subsurface iron on the octahedral $\mathrm{Fe}(B)$ termination is sufficient to explain the $(\sqrt{2} \times$ $\sqrt{2}) R 45^{\circ}$ surface reconstruction observed in LEED experiments $[8,10]$. Our studies do not exclude modification of the surface stoichiometry under UHV conditions; however, the surface reconstruction mechanism based on charge or orbital ordering is simpler, as it does not require mass transport. Stoichiometry modification can be perceived as a local effect. The high resolution STM experiments already provide evidence that entities similar to atomic dimers are present on the surface $[8,10]$.

The surface sensitive electron spectroscopic studies of magnetite suggest semiconducting character of magnetite below and above $T_{V}[33,34]$, and they also reveal only partial $(\sim 60 \%)$ spin polarization around the Fermi level [35]. Since the penetration depth of those techniques extends dozens of angstroms, the observed average spectra contains both occupied surface states $\left(\sim 0.5 \mathrm{eV}\right.$ below $\left.E_{F}\right)$ and empty valence band features of the bulk; thus, $100 \%$ spin polarization is not seen (see Fig. 2(c)). However, the theoretical surface depth profile of the orbital ordering remains to be investigated in the future.

To summarize, we report DFT studies of the (001) surface of magnetite that account for the on-site Coulomb interaction of $\mathrm{Fe} d$ electrons. Our calculations provide evidence for charge and $t_{2 g}$ orbital ordering in the first two surface layers, which is present for various surface stoichiometries. The charge ordering opens the band gap at the surface, and it is responsible for the $(\sqrt{2} \times \sqrt{2}) R 45^{\circ}$ surface reconstruction for the octahedral termination.

Fruitful discussions with Professor J. Korecki and Dr. J. Toboła are kindly acknowledged. The support of Polish Ministry of Sciences and Higher Education for the Project No. N202 119 31/1792 in the years 2006-2008 and CPU time allocation on MareNostrum, BSC supercomputer are kindly acknowledged.

\footnotetext{
*Zbigniew.Lodziana@empa.ch
}

[1] E. J. W. Verwey, Nature (London) 144, 327 (1939).

[2] J. B. Carlson, Science 189, 753 (1975).

[3] F. Walz, J. Phys. Condens. Matter 14, R285 (2002).

[4] J. Garcia and G. Subias, J. Phys. Condens. Matter 16, R145 (2004).

[5] P. W. Anderson, Phys. Rev. 102, 1008 (1956).

[6] I. Leonov et al. Phys. Rev. Lett. 93, 146404 (2004).

[7] H.-T. Jeng, G. Y. Guo, and D. J. Huang, Phys. Rev. Lett. 93, 156403 (2004).

[8] N. Spiridis, J. Barbasz, Z. Łodziana, and J. Korecki, Phys. Rev. B 74, 155423 (2006).

[9] K. Jordan et al., Phys. Rev. B 74, 085416 (2006).

[10] R. Wiesendanger et al., Science 255, 583 (1992).

[11] S. A. Chambers, S. Thevuthasan, and S. A. Joyce, Surf. Sci. 450, L273 (2000).

[12] G. Subías et al., Phys. Rev. Lett. 93, 156408 (2004).

[13] E. Nazarenko et al., Phys. Rev. Lett. 97, 056403 (2006).

[14] P. Piekarz, K. Parlinski, and A. M. Oleś, Phys. Rev. Lett. 97, 156402 (2006).

[15] H. Seo, M. Ogata, and H. Fukuyama, Phys. Rev. B 65, 085107 (2002).

[16] I. Leonov and A. N. Yaresko, J. Phys. Condens. Matter 19, 021001 (2007).

[17] B. Stanka, W. Hebenstreit, U. Diebold, and S. A. Chambers, Surf. Sci. 448, 49 (2000).

[18] C. Noguera, J. Phys. Condens. Matter 12, R367 (2000).

[19] R. Pentcheva et al., Phys. Rev. Lett. 94, 126101 (2005).

[20] S. L. Dudarev, G. A. Botton, S. Y. Savrasov, C. J. Humphreys, and C.J. Sutton, Phys. Rev. B 57, 1505 (1998).

[21] G. Kresse and J. Furthmüller, Comput. Mater. Sci. 6, 15 (1996).

[22] G. Kresse and D. Joubert, Phys. Rev. B 59, 1758 (1999).

[23] J. P. Perdew et al., Phys. Rev. B 46, 6671 (1992).

[24] Calculations with $U=4.0,5.5$ or $J=0.5,1.0$ give the same electronic effects for the octahedral $\mathrm{Fe}(B)$ surface. Lack of the on-site interaction $U=J=0.0$ leads to surface metallization. The numerical value of the band gap weakly depends on the on-site parameters [36]; details will be published elsewhere [30].

[25] O. Bengone, M. Alouani, P. Blöchl, and J. Hugel, Phys. Rev. B 62, 16392 (2000).

[26] K. Reuter and M. Scheffler, Phys. Rev. B 65, 035406 (2001).

[27] Z. Łodziana, J. K. Nørskov, and P. Stoltze, J. Chem. Phys. 118, 11179 (2003).

[28] C. Cheng, Phys. Rev. B 71, 052401 (2005).

[29] C. Noguera, A. Pojani, P. Casek, and F. Finocchi, Surf. Sci. 507-510, 245 (2002).

[30] Z. Łodziana (to be published).

[31] Z. Szotek et al., Phys. Rev. B 68, 054415 (2003).

[32] V. N. Antonov et al., Phys. Rev. B 64, 134410 (2001).

[33] J.-H. Park et al., Phys. Rev. B 55, 12813 (1997).

[34] D. Schrupp et al., Europhys. Lett. 70, 789 (2005).

[35] D. J. Huang et al., J. Magn. Magn. Mater. 239, 261 (2002).

[36] H. P. Pinto and S. D. Elliott, J. Phys. Condens. Matter 18, 10427 (2006). 